\title{
Clostridium difficile colitis following treatment with metronidazole and vancomycin
}

\author{
P.J. Bingley and G.M. Harding \\ Department of Medicine, Whittington Hospital, Highgate Hill, London N19 5NF, UK.
}

\begin{abstract}
Summary: A 25 year old woman developed Clostridium difficile colitis following a course of vancomycin and metronidazole prescribed for pelvic inflammatory disease. The condition resolved after treatment with vancomycin given alone. Colitis following this combination of antibiotics has not been described previously.
\end{abstract}

\section{Introduction}

Clostridium difficile colitis is a recognized complication of treatment with a variety of antibiotics.' Vancomycin and metronidazole have both been recommended in the management of the disease. Metronidazole given alone has been implicated in its development ${ }^{2}$ and a persistent carrier state has been reported following vancomycin therapy. ${ }^{3}$ However we believe that this is the first report of the condition developing whilst taking this combination of drugs.

\section{Case report}

A 25 year old secretary was admitted with a 10-day history of nausea, anorexia, peri-umbilical pain and bloody diarrhoea. She was passing blood and mucus per rectum 12 times daily with 2 watery brown stools per day. There was no history of previous gastrointestinal disturbance. Two weeks prior to the onset of symptoms she had been prescribed metronidazole tablets $1500 \mathrm{mg}$ /day and vancomycin capsules $500 \mathrm{mg} /$ day for pelvic inflammatory disease and had completed a 14 day course of treatment. There had been no other exposure to antibiotics.

On examination she was apyrexial but looked unwell. There was tenderness to deep palpation in the left iliac fossa. Rectal examination was normal with no peri-anal disease. Sigmoidoscopy to $15 \mathrm{~cm}$ showed an erythematous mucosa with mucus and liquid brown stool. There was no blood or contact bleeding. No pseudomembranes were seen.

Full blood count and biochemistry were normal. Stool microscopy showed moderate numbers of red

Correspondence: P. Bingley, M.B., M.R.C.P. Department of Diabetes and Immunogenetics, St. Bartholomew's Hospital, West Smithfield, London EC1 7BE, UK

Accepted: 30 April 1987 cells only and culture was negative. On rectal biopsy predominantly chronic inflammatory changes in the lamina propria with some acute inflammation with one small crypt abscess were seen. Barium enema demonstrated only a rather granular appearance of the mucosa in the rectum and sigmoid. $C$. difficile toxin was detected in the stool.

The patient continued to have frequent bloody diarrhoea throughout the course of her investigations. She was eventually commenced on vancomycin $500 \mathrm{mg} /$ day and within 4 days her bowel habit had returned to normal. Repeated examination of the stool for $C$. difficile toxin was negative after treatment.

\section{Discussion}

Antibiotic-associated colitis is thought to be due to depletion of gut flora allowing proliferation of $C$. difficile and its production of a cytopathic endotoxin which induces necrotizing lesions in the colonic mucosa. The presence of a faecal toxin neutralized by C. sordellii is diagnostic. ${ }^{1}$

Treatment of the condition with vancomycin is usually effective causing elimination of both $C$. difficile and its toxin. Relapses after vancomycin therapy have been reported, ${ }^{4}$ being attributed to the fact that spores may survive the customary 5 day course and germinate once the treatment has been completed. Metronidazole is more efficiently absorbed from the gastrointestinal tract leaving only a small proportion of any dose remaining in the gut lumen. This is probably the cause of the relatively frequent failure of treatment with this drug.

The reason for the permissive effect of the combination of these antibiotics on the growth of $C$. difficile is not clear. It cannot be explained by relative resistance

(C) The Fellowship of Postgraduate Medicine, 1987 
of the $C$. difficile organisms to vancomycin in view of the rapid clinical response and resolution of toxin production when vancomycin alone was given. Both antibiotics are bactericidal, vancomycin acting by inhibiting peptido-glycan formation and metronidazole by damaging DNA. Thus it is unlikely that the two drugs have any mutually inhibiting effect. Faecal anaerobes have been shown to be noticeably suppressed in patients with pseudomembranous colitis ${ }^{5}$ and metronidazole has high bacterial activity against

\section{References}

1. Anonymous. Antibiotic-associated colitis - the continuing saga. $\mathrm{Br}$ Med J 1981, 282: 1913-1914.

2. Sagimer, R., Hawley, C.R. \& Bartlett, J.G. Colitis associated with metronidazole therapy. J Infect Dis 1980 , 141: $772-774$.

3. Bartlett, J.G., Tedesco, F.J., Shull, S., Lowe, B. \& Chang, T. Symptomatic relapse after vancomycin therapy of antibiotic-associated pseudomembranous colitis. Gastroenterology 1980, 78: 431-434. anaerobic organisms. It is therefore presumed that the $\frac{\pi}{\infty}$ overgrowth of $C$. difficile made possible by the depletion of anaerobes resulting from metronidazole ${ }^{c} \cdot$ therapy outweighed the effect of vancomycin in eliminating the pathogenetic organism. When metronidazole was withdrawn an element of inhibition of the organism by normal intestinal flora must have been restored, enabling vancomycin to exert its bactericidal effect on the $C$. difficile organisms.

4. Finch, R.G., McKim Thomas, H.J., Lewis, M.T., Slack R.C.B. \& George, W.L. Relapse of pseudomembranouscolitis after vancomycin therapy. Lancet 1979, ii: 1076-C. 1077.

5. Kappas, A., Shinagawa, N., Arabi, Y. et al. Diagnosis of pseudomembranous colitis. $\mathrm{Br}$ Med J 1978, i: 675-676. 